\title{
Cardiac-gated intracranial elastance in a swine model of raised intracranial pressure: a novel method to assess intracranial pressure-volume dynamics
}

\author{
Omer Doron, MD, ${ }^{1,2}$ Ofer Barnea, PhD, ${ }^{2}$ Nino Stocchetti, MD, ${ }^{3}$ Tal Or, MSc, ${ }^{2}$ Erez Nossek, MD, ${ }^{4}$ and \\ Guy Rosenthal, MD'
}

\begin{abstract}
1Department of Neurosurgery, Hadassah-Hebrew University Medical Center, Jerusalem; '2Department of Biomedical Engineering, Tel Aviv University, Tel Aviv, Israel; ${ }^{3}$ Department of Physiopathology and Transplantation, Milan University and Neuro ICU Fondazione IRCCS Cà Granda Ospedale Maggiore Policlinico, Milan, Italy; and ${ }^{2}$ Department of Neurosurgery, New York University Medical Center, New York, New York
\end{abstract}

\begin{abstract}
OBJECTIVE Previous studies have demonstrated the importance of intracranial elastance; however, methodological difficulties have limited widespread clinical use. Measuring elastance may offer potential benefit in helping to identify patients at risk for untoward intracranial pressure (ICP) elevation from small rises in intracranial volume. The authors sought to develop an easily used method that accounts for the changing ICP that occurs over a cardiac cycle and to assess this method in a large-animal model over a broad range of ICPS.
\end{abstract}

METHODS The authors used their previously described cardiac-gated intracranial balloon pump and swine model of cerebral edema. In the present experiment they measured elastance at 4 points along the cardiac cycle-early systole, peak systole, mid-diastole, and end diastole-by using rapid balloon inflation to $1 \mathrm{ml}$ over an ICP range of 10-30 $\mathrm{mm} \mathrm{Hg}$.

RESULTS The authors studied 7 swine with increasing cerebral edema. Intracranial elastance rose progressively with increasing ICP. Peak-systolic and end-diastolic elastance demonstrated the most consistent rise in elastance as ICP increased. Cardiac-gated elastance measurements had markedly lower variance within swine compared with noncardiac-gated measures. The slope of the ICP-elastance curve differed between swine. At ICP between 20 and $25 \mathrm{~mm}$ $\mathrm{Hg}$, elastance varied between 8.7 and $15.8 \mathrm{~mm} \mathrm{Hg} / \mathrm{ml}$, indicating that ICP alone cannot accurately predict intracranial elastance.

CONCLUSIONS Measuring intracranial elastance in a cardiac-gated manner is feasible and may offer an improved precision of measure. The authors' preliminary data suggest that because elastance values may vary at similar ICP levels, ICP alone may not necessarily best reflect the state of intracranial volume reserve capacity. Paired ICP-elastance measurements may offer benefit as an adjunct "early warning monitor" alerting to the risk of untoward ICP elevation in brain-injured patients that is induced by small increases in intracranial volume.

https://thejns.org/doi/abs/10.3171/2020.3.JNS193262

KEYWORDS cardiac-gated elastance; cerebral edema; intracranial balloon pump; intracranial pressure; swine model; traumatic brain injury

$\mathrm{T}$ HE intracranial pressure-volume (PV) relationship first hypothesized by Monro and Kellie serves as the basis for our understanding of intracranial pressure (ICP) dynamics. ${ }^{1,2}$ The total intracranial volume is a sum of its 3 components: brain, blood, and CSF, all of which are enclosed by the dura mater with its limited elastance and the rigid cranium. As such, it has long been understood that the intracranial PV relationship is exponential-like, with a restricted ability to tolerate increases in intracranial volume before a rapid rise in ICP results. In clinical practice, estimating when the limit of intracranial volume reserve capacity has been reached is of crucial importance in guiding medical and surgical decisions in the neurointensive care unit. Basing an estimate of the intracranial volume reserve capacity on ICP alone, although routinely done, has theoretical limitations, because the same ICP may reflect different positions on the intracranial PV curve (Fig. 1). Measuring elastance in brain-injured

ABBREVIATIONS ICP = intracranial pressure; $\mathrm{PV}=$ pressure-volume; $\mathrm{TBI}$ = traumatic brain injury.

SUBMITTED December 3, 2019. ACCEPTED March 31, 2020.

INCLUDE WHEN CITING Published online June 5, 2020; DOI: 10.3171/2020.3.JNS193262. 

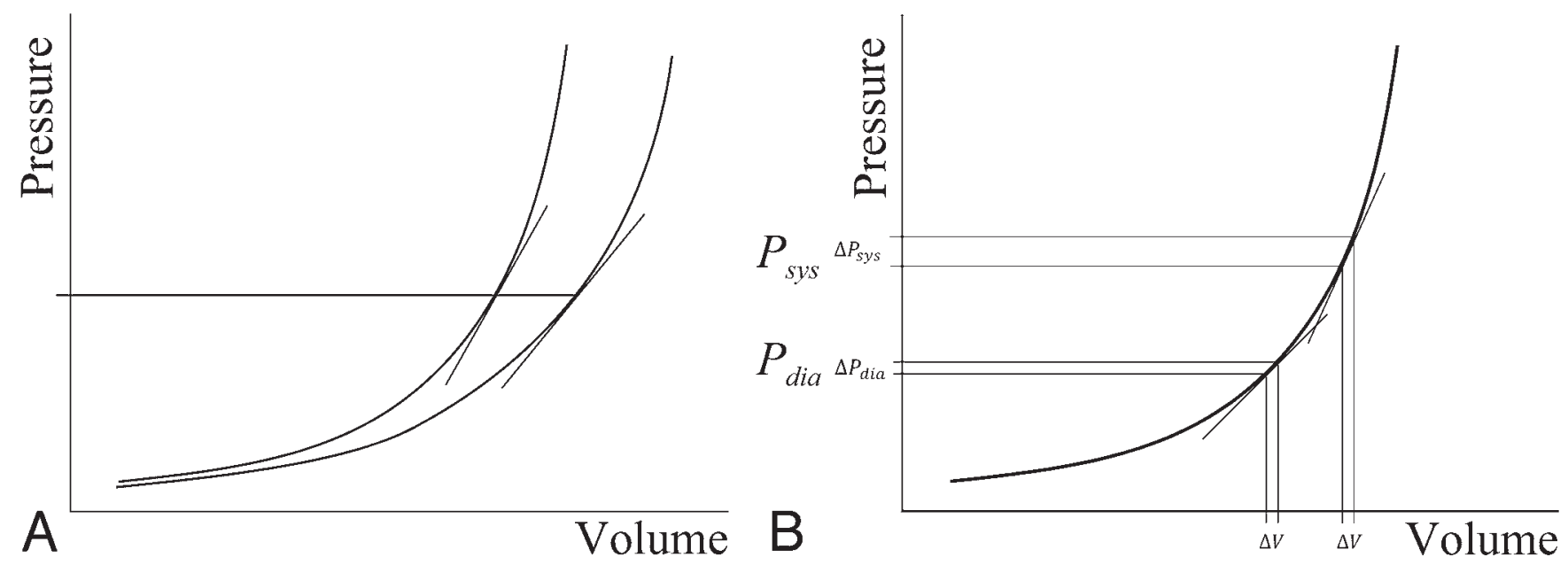

FIG. 1. A: Theoretical PV curves in different subjects. With a left or right shift of the curve and with differing slopes, subjects with the same ICP may have different intracranial elastance. B: The PV curve demonstrates how measured elastance may differ between diastole and systole. The same volume perturbation leads to a different pressure response at diastole than at systole, suggesting a potential benefit of obtaining elastance measurements in a fashion that is gated to the cardiac cycle. Elastance at diastole and systole is calculated as the change in pressure at diastole $\left(\Delta \mathrm{P}_{\text {dia }}\right)$ or systole $\left(\Delta \mathrm{P}_{\text {sys }}\right)$, respectively, divided by the change in volume $(\Delta \mathrm{V})$ that induces the pressure rise.

patients has the potential to identify those who are at risk for deterioration precipitated by a small increase in intracranial volume.

Intracranial elastance, defined as the relationship between a given change in pressure and a given change in volume, can be measured in both the experimental and clinical setting. Pioneering work by led by Langfitt, Marmarou, Miller, and their respective colleagues described this relationship and defined key concepts such as the volume pressure index (VPI) and volume pressure response (VPR). ${ }^{3-7}$ These early studies demonstrated that the slope of the intracranial PV curve varies between subjects. ${ }^{8,9}$ The authors stressed the importance of measuring intracranial elastance in individual patients to gain insight into the state of the intracranial volume reserve capacity. ${ }^{6,10}$ However, these works performed mostly in the 1970s and 1980s were limited by intermittent measurements performed either by manual injection or at relatively slow constant infusion rates and could not account for the constant changes in intracranial volume that occur with every heartbeat. ${ }^{11,12}$ The volume changes that occur as arterial blood is injected into the cranium during each cardiac cycle and the corresponding exit of venous blood and CSF leaving the cranium are significant and are the basis of the ICP waveform. ${ }^{13}$

To date, no method used to measure intracranial elastance has taken into account these PV changes related to the cardiac cycle. In addition, many of the classic animal studies of intracranial elastance relied on the injection of progressively increasing volumes from the same baseline ICP, and therefore did not accurately estimate elastance as a function of raised ICP and increasing brain edema. We hypothesized that the volume changes occurring with each cardiac cycle affect the PV relationship and influence measured elastance at different ICP levels (Fig. 1). We sought to develop a method to measure intracranial elastance in a cardiac-gated fashion over a broad range of ICPs in a large-animal model of cerebral edema and to ascertain the potential advantages of measuring elastance in this manner.

\section{Methods}

\section{Preparation of the Animal}

We studied female Yorkshire swine weighing 38-42 $\mathrm{kg}$ each in the large-animal sterile Experimental Surgical Facility of the Hadassah-Hebrew University Medical School. Swine were obtained from a licensed supplier (Institute for Animal Research), quarantined for a minimum of 7 days and maintained in our accredited animal care facility. The study was approved by the institutional review board, and animal care complied with the Guide for the Care and Use of Laboratory Animals.

Swine were anesthetized and prepared as previously reported.${ }^{14} \mathrm{ECG}$, arterial blood pressure, respiration, temperature, and arterial blood gases were monitored. Blood gas values were maintained within normal range $(\mathrm{pH}$ 7.35-7.45, $\mathrm{PaCO}_{2} 35-45 \mathrm{~mm} \mathrm{Hg}, \mathrm{PaO}_{2} 90-105 \mathrm{~mm} \mathrm{Hg}$ ) by controlling ventilation volume and frequency, while body temperature was maintained with a thermal blanket $\left(37^{\circ} \mathrm{C}\right.$ $\pm 3^{\circ} \mathrm{C}$ ). After exposure of the skull, 3 separate burr holes were drilled using an automatic drill (Summex; Stryker). The holes were located $1 \mathrm{~cm}$ left of midline and $1 \mathrm{~cm}$ anterior to the coronal suture, $1 \mathrm{~cm}$ left of midline and $1 \mathrm{~cm}$ posterior to the coronal suture, and $1 \mathrm{~cm}$ right of midline and $1 \mathrm{~cm}$ posterior to the coronal suture. An intraparenchymal ICP monitor (Mikro-Tip pressure catheter; Millar) was inserted into the parenchyma to a depth of 1-1.5 $\mathrm{cm}$ through the left anterior burr hole. The intracranial balloon used to measure elastance was inserted through the left posterior burr hole and placed into the ventricle under ultrasound guidance. We inserted a 16-gauge continuous-drainage catheter (Codman Neuro USA) through the right posterior burr hole into the ventricle until clear 


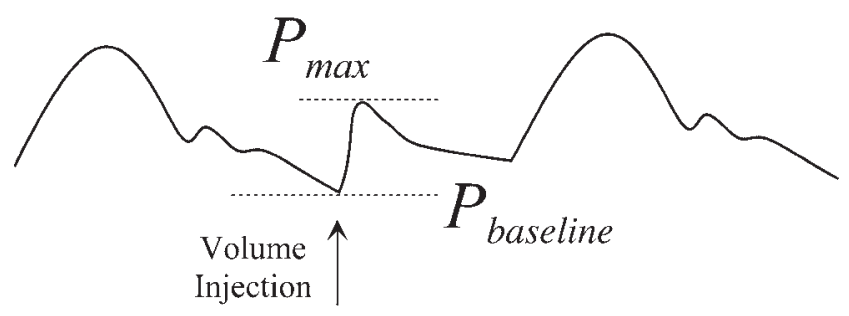

FIG. 2. ICP waveform that demonstrates a pressure response to a rapid volume change induced by balloon inflation. $P_{\max }$ denotes the value of peak ICP response, and $P_{\text {baseline }}$ is the ICP value just prior to balloon inflation.

CSF was seen in the lumen. All burr holes were closed with bone wax.

\section{Intracranial Balloon Pump and Experimental Protocol}

The experimental system is composed of a linear motor that rapidly drives a syringe pump connected to a balloon via a high-pressure catheter and has been described in detail in a previous work..$^{15}$ The intracranial balloon pump device was used to measure cardiac-gated elastance, first at baseline ICP and then sequentially with every $5-\mathrm{mm}$ $\mathrm{Hg}$ increment in ICP. The intracranial balloon pump was activated intermittently for a period of approximately 10 seconds at each ICP level. Activation was performed 25-30 times at each ICP level in order to assess variance between measures. Elastance was measured at fixed time points along the cardiac cycle. As described in a previous work, we raised ICP by intravenous infusion of hypotonic solution $(0.18 \% \mathrm{NaCl}+\mathrm{D} 5 \mathrm{~W})$ to induce hyponatremia and intracellular cerebral edema. ${ }^{14}$ This model of water intoxication leads to a graded and sustained rise in ICP as cerebral edema worsens.

As seen in Fig. 2, we calculated intracranial elastance (E) as the ratio of the difference of the immediate rise in ICP post-balloon inflation $\left(\mathrm{P}_{\max }\right)$ and pre-balloon inflation $\left(\mathrm{P}_{\text {baseline }}\right)$, divided by the volume of balloon inflation $(\Delta \mathrm{V}=1 \mathrm{ml}): \mathrm{E}=\mathrm{P}_{\max }-\mathrm{P}_{\text {baseline }} / \Delta \mathrm{V}$. We calculated elastance at different time points along the cardiac cycle in relation to the $\mathrm{R}$ wave on ECG. At each ICP level, inflation was timed to occur precisely at the same part of the cardiac cycle, followed by balloon deflation 300-500 msec afterward. Elastance values were calculated as an average over 50 cardiac cycles and classified according to their temporal location relative to the cardiac cycle. As seen in Fig. 3, at each ICP level, elastance was calculated at 4 time points along the cardiac cycle: early systole $\left(\mathrm{t}_{1}\right)$, peak systole $\left(t_{2}\right)$, mid-diastole $\left(t_{3}\right)$, and end diastole $\left(t_{4}\right)$. Elastance was calculated as the mean of 50 inflation-deflation cycles at each point along the cardiac cycle. In order to ascertain whether cardiac-gated elastance differs from elastance not gated to the cardiac cycle, we also calculated a lumped intracranial elastance taken from random points along the cardiac cycle.

\section{Data Processing}

Data were acquired at a rate of $200 \mathrm{~Hz}$ and analyzed using an automated algorithm that we developed on MATLAB (R2014b, MathWorks). For waveform analysis, ICP
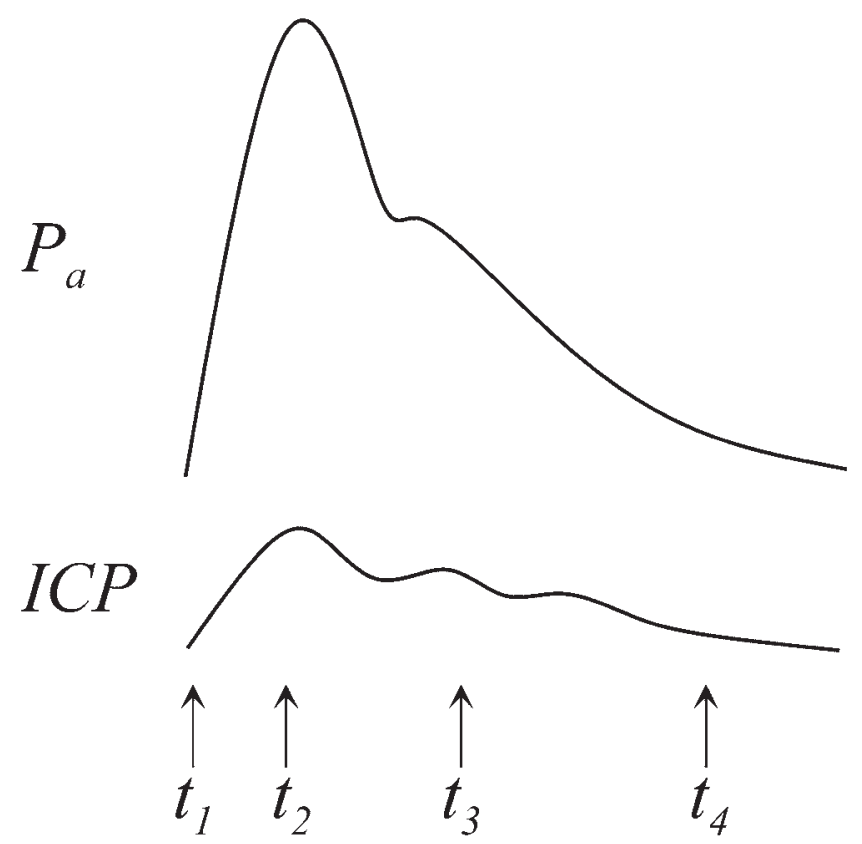

FIG. 3. Method used to measure intracranial elastance in a cardiacgated elastance fashion. The upper waveform represents the arterial waveform over one cardiac cycle $\left(P_{\mathrm{a}}\right)$. The lower waveform represents the ICP waveform over the same cardiac cycle. Four arrows represent 4 points in time relative to the cardiac cycle in which the inflation-deflation of a balloon takes place. These points are as follows: $t_{1}$, early systole; $t_{2}$, peak systole; $t_{3}$, mid-diastole; and $t_{4}$, end diastole. Every inflation-deflation cycle is synchronous with a different portion of the cardiac cycle, yielding a specific elastance value for that time point that is equal to the derivative $\Delta \mathrm{P} / \Delta \mathrm{V}$.

and arterial carotid pressure were high-pass filtered to isolate the cardiac-related variations from respiratory and other slow waves. All estimations of ICP and elastance were performed on unfiltered raw data at $200 \mathrm{~Hz}$.

\section{Statistical Analysis}

Data were analyzed using a mixed-design ANOVA model to account for repeated measures in each animal. Data were analyzed to assess whether statistically significant differences in cardiac-gated measures of elastance existed between elastance measured at different ICP levels. Similarly, we used the mixed-design ANOVA to assess differences in the variance of measure within swine at different ICP levels and at different points along the cardiac cycle.

\section{Results}

We studied 7 swine at progressively increasing levels of ICP and measured cardiac-gated elastance at each of 4 points along the cardiac cycle. In general, cardiac-gated elastance increased progressively as brain edema increased and ICP rose from baseline to peak levels (Fig. 4). This progressive increase was most evident at peak systole $\left(t_{2}\right)$ and end diastole $\left(t_{4}\right)$, where the rise in elastance is consistent at each progressively increasing level of ICP, indicating that these points may best reflect the state of intracranial compliance. In contrast, at early systole $\left(t_{1}\right)$ 


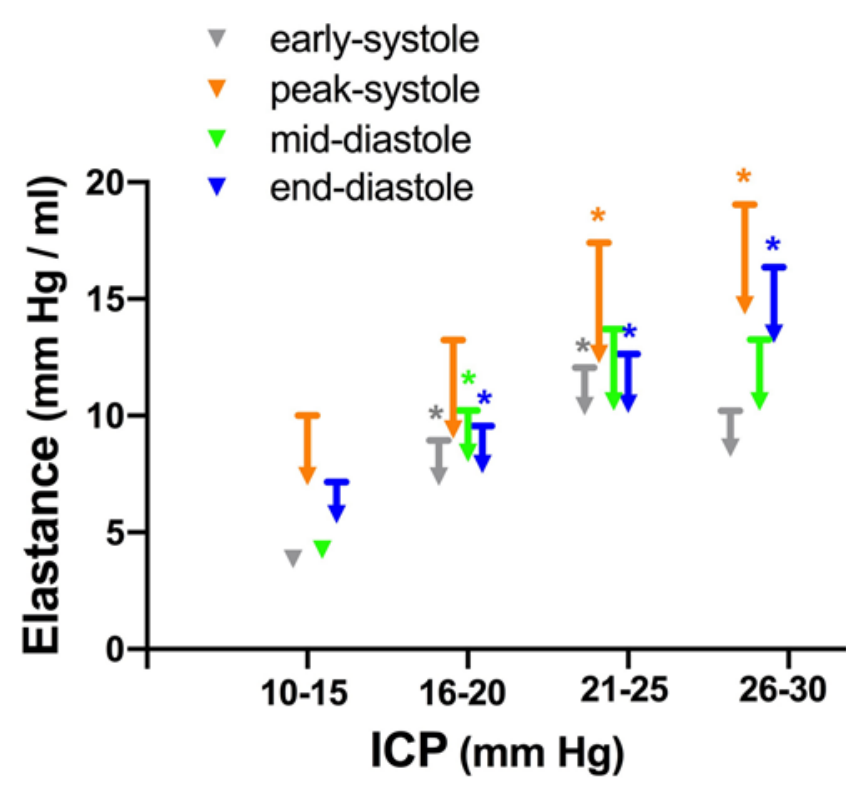

FIG. 4. Intracranial cardiac-gated elastance measured at different ICP levels. A consistent increase in intracranial cardiac-gated elastance is seen as brain swelling increases and ICP rises. As expected, peak systolic elastance is greater than elastance at other points along the cardiac cycle because peak systole represents the point at which intracranial volume and pressure are at their greatest. Both peak systole and end diastole show a consistent rise in measured elastance at progressively higher ICP levels. ${ }^{*} p<0.05$, assessed by mixed-design ANOVA denoting a significant difference in comparison to the mean cardiac-gated elastance value at the previous ICP level.

and mid-diastole $\left(\mathrm{t}_{3}\right)$, elastance reached a plateau at 20-25 $\mathrm{mm} \mathrm{Hg}$ and did not rise further beyond that point. In addition, gating elastance measurements to the cardiac cycle revealed important differences between different points along the cardiac cycle. As expected, elastance measurements at peak systole were greater than at all other points (Fig. 4), because the intradural compartment attains its highest volume state at that point.

We sought to compare cardiac-gated elastance to a non-cardiac-gated, or lumped, elastance measure. Lumped intracranial elastance also increased progressively with increasing brain edema and rising ICP, reaching a plateau at $20-25 \mathrm{~mm} \mathrm{Hg}$, above which it did not increase further (Table 1). While the variance of lumped elastance was similar to cardiac-gated elastance across swine, this was not the case within individual swine, where the vari-

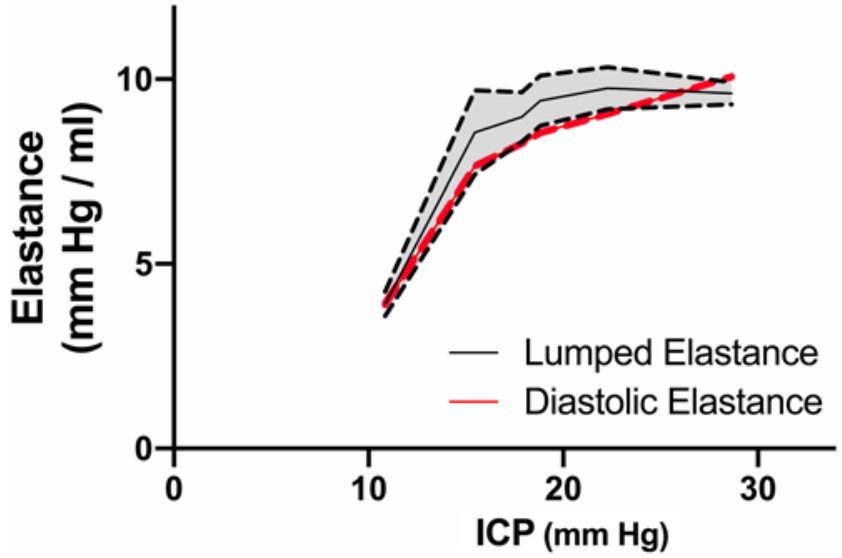

FIG. 5. The relationship of lumped intracranial elastance (gray line) and end-diastolic intracranial elastance (red line) with ICP in a single swine. The shaded gray region demonstrates the bounds of 1 SD of the measured lumped intracranial elastance in the swine. The thickened portion of the red line indicates the standard deviation of end-diastolic measure of intracranial elastance. The variance of measure is at least one order of magnitude greater in the lumped intracranial elastance measure when compared to the end-diastolic intracranial elastance measure. The decreased variance of the cardiac-gated end-diastolic measure suggests a potential benefit of the cardiac-gated method that could improve the precision of elastance estimation in individual subjects.

ance in measured elastance was substantially lower when gated to the cardiac cycle (Fig. 5). As seen in Table 2, for all points along the cardiac cycle and at all ICP values the variance of elastance measurements within swine was reduced approximately 10-fold when compared to the mean variance of a lumped measure of intracranial elastance within swine, indicating a considerably improved precision in measurement and the potential to better define the actual intracranial volume reserve capacity in individual subjects.

Figure 6 demonstrates end-diastolic elastance values across ICP in all swine. We chose the end-diastolic measure of elastance because it demonstrated a consistent rise as cerebral edema and ICP increased. As seen in Fig. 6, our preliminary data suggest that the ICP-elastance relationship differs between swine. Importantly, at similar ICP levels, different swine had differing elastance values and the rate of change in elastance as ICP increased also varied between swine. The ICP-elastance curve may be shifted leftward or rightward between swine and its slope is variable, potentially indicating that ICP alone cannot

TABLE 1. Physiological parameters and lumped intracranial elastance measured at different ICP ranges

\begin{tabular}{lcccc}
\hline & \multicolumn{4}{c}{ ICP Level } \\
\cline { 2 - 5 } Parameter & $10-15 \mathrm{~mm} \mathrm{Hg}$ & $16-20 \mathrm{~mm} \mathrm{Hg}$ & $21-25 \mathrm{~mm} \mathrm{Hg}$ & $26-30 \mathrm{~mm} \mathrm{Hg}$ \\
\hline Heart rate $(\mathrm{bpm})$ & $70 \pm 5$ & $72 \pm 7$ & $70 \pm 7$ & $76 \pm 8$ \\
\hline Mean arterial pressure $(\mathrm{mm} \mathrm{Hg})$ & $70 \pm 5$ & $68 \pm 5$ & $11.0 \pm 2.91$ & $11.9 \pm 2.85^{*}$ \\
\hline Lumped intracranial elastance $(\mathrm{mm} \mathrm{Hg} / \mathrm{ml})$ & $5.32 \pm 1.08$ & $8.28 \pm 2.21$ & & $72 \pm 4$ \\
\hline
\end{tabular}

All values are expressed as the mean $\pm S D$.

* Mixed-design ANOVA reveals a significant difference $(p<0.05)$ between lumped intracranial elastance at the 4 ICP ranges. However, the mixed-design ANOVA did not demonstrate a statistically significant difference in lumped intracranial elastance when sequential ICP ranges were compared. 
TABLE 2. The mean variance of measured cardiac-gated and lumped intracranial elastance in individual swine at different ICP ranges

\begin{tabular}{|c|c|c|c|c|}
\hline \multirow[b]{2}{*}{ Intracranial Elastance } & \multicolumn{4}{|c|}{ ICP Level } \\
\hline & $10-15 \mathrm{~mm} \mathrm{Hg}$ & $16-20 \mathrm{~mm} \mathrm{Hg}$ & $21-25 \mathrm{~mm} \mathrm{Hg}$ & $26-30 \mathrm{~mm} \mathrm{Hg}$ \\
\hline \multicolumn{5}{|l|}{ Early systole } \\
\hline Mean variance of measure in individual swine & 0.06 & 0.03 & 0.02 & 0.03 \\
\hline \multicolumn{5}{|l|}{ Peak systole } \\
\hline Mean variance of measure in individual swine & 0.02 & 0.01 & 0.01 & 0.01 \\
\hline \multicolumn{5}{|l|}{ Mid-diastole } \\
\hline Mean variance of measure in individual swine & 0.04 & 0.02 & 0.01 & 0.02 \\
\hline \multicolumn{5}{|l|}{ End diastole } \\
\hline Variance of measure in individual swine & 0.03 & 0.01 & 0.01 & 0.01 \\
\hline \multicolumn{5}{|l|}{ Lumped intracranial elastance } \\
\hline Variance of measure in individual swine & $0.55^{\star}$ & $0.77^{*}$ & $0.66^{*}$ & $0.60^{*}$ \\
\hline
\end{tabular}

Within individual swine, the variance in the measure of cardiac-gated intracranial elastance at each point along the cardiac cycle and at all ICP ranges is low. In contrast, the variance of measure in a lumped measure of intracranial elastance in individual swine is significantly greater at all ICP ranges when compared to the variance of the cardiac-gated measure of intracranial elastance.

${ }^{*} p<0.001$.

accurately predict intracranial elastance and that intracranial volume buffering capacity may differ between swine. At the clinically important threshold of ICP between 20 and $25 \mathrm{~mm} \mathrm{Hg}$, end-diastolic elastance varied between 8.7 and $15.8 \mathrm{~mm} \mathrm{Hg} / \mathrm{ml}$, indicating that in this ICP range the increase in pressure caused by a small increase in intracranial volume can vary nearly 2 -fold. Conversely, an enddiastolic elastance threshold of $8 \mathrm{~mm} \mathrm{Hg} / \mathrm{ml}$ was reached between a wide range of ICPs (13-22 $\mathrm{mm} \mathrm{Hg}$ ), again indicating that ICP alone may not accurately predict intracranial volume reserve capacity.

\section{Discussion}

In this study we demonstrate that measuring elastance in a cardiac-gated fashion is feasible in a large-animal

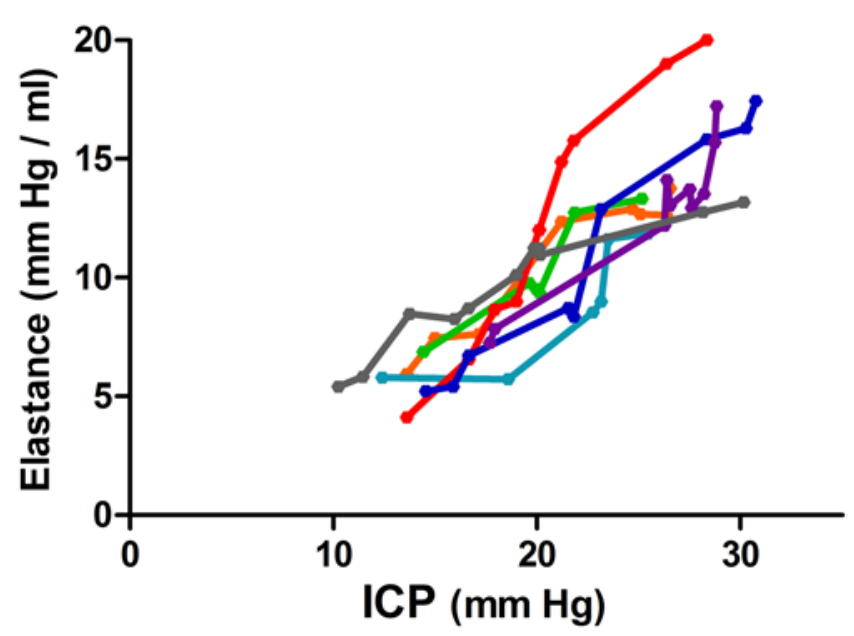

FIG. 6. The relationship between end-diastolic elastance and ICP in all 7 swine. The slope and position (left or right shift) of the elastance-ICP curve vary substantially between swine, indicating that ICP alone does not necessarily predict the intracranial reserve capacity between swine. At ICP values in the clinically significant range of $20-25 \mathrm{~mm} \mathrm{Hg}$, measured elastance varies substantially, from 8.7 to $15.8 \mathrm{~mm} \mathrm{Hg} / \mathrm{ml}$. model. As expected, our results clearly indicate a substantial increase in elastance with worsening brain edema and increased ICP. We also demonstrate the effect of timing measures of intracranial elastance to the cardiac cycle. To our knowledge, previous investigations have not systematically studied measures of intracranial elastance in a cardiac-gated fashion in large animals or in patients. As anticipated, our results demonstrate differences in elastance between different points on the cardiac cycle. Not surprisingly, peak systolic elastance is consistently greater than elastance measured at other points on the cardiac cycle across all ranges of ICP. This makes sense, because intracranial volume and pressure are at their peak during peak systole. Importantly, both peak-systolic and enddiastolic elastance show the most consistent increase in elastance values as cerebral swelling increases and ICP rises, indicating that measuring elastance at one of these two points may offer the most clinically meaningful assessment of intracranial volume reserve (Fig. 4). In theory, end-diastolic elastance has the advantage of being an optimal time point to measure elastance because it is the point at which the intracranial contents are at their most "relaxed" state, with the majority of venous blood exiting the cranium and a minimum of arterial inflow. Further work is needed to confirm which time points relative to the cardiac cycle provide the most meaningful information regarding the state of intracranial volume reserve.

Elastance, defined as the change in pressure divided by the change in volume $(\Delta \mathrm{P} / \Delta \mathrm{V})$, is the inverse of compliance, which is defined as the change in volume divided by the change in pressure $(\Delta \mathrm{V} / \Delta \mathrm{P})$. Both elastance and compliance assess the relationship between changes in volume and pressure along the PV curve. Pioneers in the field disagreed on whether to use a measure of compliance or elastance. Marmarou et al. promoted a compliance-based measure, ${ }^{5}$ whereas Leech and Miller advocated for a measure based on elastance. ${ }^{16}$ Leech and Miller argued that for the clinician, elastance was the more easily grasped parameter because, like ICP, it rises as the state of intracranial volume reserve worsens. We support the view that 
elastance may be a more intuitive parameter for the clinician.

Measuring elastance in brain-injured patients may, in principle, help to predict which ones are at risk for sudden deterioration due to an untoward rise in ICP induced by a small change in volume. Our current study suggests that this may be achieved with brief intermittent activation of the cardiac-gated intracranial balloon lasting for only several seconds, which may be advantageous from the perspective of patient safety if this technology is translated into clinical use. Several pioneering studies demonstrated the potential clinical utility of measuring intracranial elastance. ${ }^{7,9,17}$ Miller and Pickard demonstrated that elastance measures may be related to the degree of midline shift in patients with traumatic brain injury (TBI). ${ }^{9}$ However, these early studies were limited by the need to use slow, constant infusion or manual injection of fluid into the intracranial space and an inability to gate measurements to the cardiac cycle.

A few recent studies have reported results obtained by measuring compliance with the Spiegelberg compliance device that inflates and deflates a balloon in the brain parenchyma to a volume of $0.2 \mathrm{~cm}^{3}$ over 6 seconds. Abdullah and colleagues report improved compliance in 12 surviving patients with TBI who underwent decompressive craniectomy. ${ }^{18}$ Piper et al. measured intracranial compliance with the Spiegelberg device in 10 patients with hydrocephalus, and found compliance values between 0.14 and $1.4 \mathrm{ml} / \mathrm{mm} \mathrm{Hg}$ (corresponding to elastance of $0.71-7.1 \mathrm{~mm}$ $\mathrm{Hg} / \mathrm{ml}) .{ }^{19}$ Other investigators have raised issues regarding the stability of compliance measurements at low ICP with the Spiegelberg device. ${ }^{20}$ A potential disadvantage of measuring intracranial compliance in this fashion is that measurements occur over a 6 -second time period and therefore cannot be gated to the cardiac cycle. Using a canine model, Anile and colleagues demonstrated that measures of intracranial compliance are time dependent. ${ }^{21}$ Although no agreed-upon standard on how to best measure intracranial elastance has been defined in the literature, it is important to keep in mind that elastance as a physical property is best defined as the instantaneous change in pressure divided by the instantaneous change in volume. As such, there is an advantage in performing balloon inflation at the most rapid rate possible.

The method we describe is of potential benefit in helping to achieve estimates of elastance that are based on very rapid changes in intracranial volume. In addition, the ability to gate elastance measures to the cardiac cycle is predicated on the ability to perform very rapid and controlled changes in intracranial volume. Our findings indicate that timing elastance measurements to the cardiac cycle is important. Cardiac-gated elastance measurements seem to better account for the changing state of elastance that occurs as the PV relationship shifts during a cardiac cycle. Within individual animals cardiac-gated elastance measures lead to substantially lower variance when compared to lumped measures of intracranial elastance taken randomly from different points along the cardiac cycle (Fig. 5). This makes sense, because the intracranial volume changes occurring through the course of a cardiac cycle lead to a shift along the PV curve, thus altering elastance. By measuring intracranial elastance at specific points along the cardiac cycle, a decrease in variance within individual swine is obtained. The observed decrease in variance is substantial, reducing the variance in measured elastance by a factor of 10 or more (Table 2). As such, the cardiac-gated method may potentially improve the precision of intracranial elastance measurements, helping to distinguish between subjects with differing intracranial volume reserve capacity. Importantly, because elastance values are reported as an average over 50 cardiac cycles, they include within them variance that may be due to fluctuations in ICP that occur with the respiratory cycle. The very low variance in the measure of intracranial elastance demonstrated in Table 2 indicates that elastance values are not substantially altered by fluctuations induced by the respiratory cycle. This finding is encouraging in implying that measures of intracranial elastance are much more strongly affected by the degree of brain edema than by normal physiological variations in ICP. An additional practical advantage to the method we describe is that measurements can be performed at any time without a need to wait for a "steady-state" or for monitor calibration.

An important finding in this study is that the relationship between ICP and elastance may differ between swine. Cardiac-gated elastance increases with increased cerebral edema and rising ICP, but the pattern and rate of rise may vary between animals. The true variability in elastance measurements between subjects will need to be better defined in larger studies. Our preliminary data indicate that the ICP-elastance curve may be shifted rightward or leftward, and its slope appears to be variable between swine (Fig. 6). If larger studies confirm these preliminary findings, it would suggest that ICP alone cannot necessarily serve as a direct correlate of intracranial elastance. In current clinical practice, ICP alone is often the best available parameter to provide the clinician with an estimate of the state of intracranial volume reserve and serves as a physiological marker for the risk of impending cerebral herniation. For this reason, an ICP threshold is often used as a trigger to initiate medical therapies or to proceed to surgical interventions including decompressive craniectomy. ${ }^{22-24}$ These therapeutic interventions aim both to decrease the ICP burden, which is known to correlate with poor outcome, ${ }^{25-27}$ and to minimize the risk of cerebral herniation that can occur with small volume increases as the intracranial volume reserve is exhausted. ${ }^{13}$

Importantly, our preliminary data indicate that the ICP threshold at which a small volume shift can lead to a large rise in ICP varies substantially between animals. Specifically, elastance at the clinically important ICP threshold of $20-25 \mathrm{~mm} \mathrm{Hg}$ varies nearly 2-fold between swine. It is possible that an elastance threshold may have clinical relevance in a manner similar to an ICP threshold. Pairing measured elastance with ICP may lead to a better estimation of where a particular patient lies on the intracranial PV curve. Many recent reviews have pointed out that ICP is "more than just a number" and should be used in a broader clinical context, rather than as a univariate parameter where treatment is based on a simple crossing-ofthreshold paradigm. ${ }^{13,28,29}$ In this regard, pairing measurements of ICP with elastance may be clinically useful. Our 
data suggest that it may be possible to use a 2-parameter concept, ICP paired with elastance, to help distinguish between subjects with differing intracranial volume reserve capacity.

The method we describe provides a relatively simple and straightforward way to obtain this additional parameter. The interaction between ICP and elastance may help to better classify patients in a manner that corresponds to their position on the PV curve and their proximity to its point of inflection. As such, it may help to tailor patientspecific protocols of care for elevated ICP and better assess the risk of herniation. Paired ICP-elastance measures could also potentially be useful in assessing the response to therapeutic measures meant to reduce cerebral edema, such as hypertonic fluid administration. Further studies to evaluate the potential benefit of paired ICP-elastance measurements obtained in a cardiac-gated fashion are warranted. If paired ICP-elastance measurements can serve as an adjunct "early warning monitor" to complement ICP monitoring in severely brain-injured patients, important clinical benefit may be achieved.

\section{Concerns Regarding Translation to Clinical Studies}

We hope that future development of this methodology will lead to studies in brain-injured patients that assess the feasibility and clinical utility of this technology. In such studies, it will be crucial to determine the safety profile for this method of measuring intracranial elastance. Importantly, in the current study we did not observe any untoward elevation in ICP in any swine at any ICP levels. However, in any clinical studies it will be imperative to determine an upper limit for elastance above which further changes in intracranial volume may induce a dangerous elevation of ICP. Defining these upper limits for intracranial elastance will be of key importance in incorporating built-in safety measures such as an automatic shutdown mode that will stop balloon inflation if predefined elastance thresholds are reached. Another potential issue that may raise concern is the issue of pressure resonance. Early studies that used balloon inflation to induce hydrocephalus in an animal model described a phenomenon of pressure resonance that may lead to increased ICP. ${ }^{30}$ However, the method of balloon inflation-deflation described in our work differs substantially because activation occurs over microseconds and the balloon is activated only intermittently over a period of several seconds and is therefore extremely unlikely to induce resonance-like effects. In any future clinical applications of this technology, potential safety concerns must remain at the forefront.

\section{Limitations of the Study}

Our study has several limitations. This is a preliminary study in a swine model of cerebral edema. Further investigations of different cerebral pathological processes including models of TBI, intracranial mass lesions, and hydrocephalus will be required to validate our work. In this exploratory research, the number of animals studied was small. Studies with a larger number of subjects will be required to better define expected values of elastance at different ICP levels and to determine variance of elastance measurements between subjects. In the current study, we observed variance in intracranial elastance between swine that we ascribe to possible differences in the intracranial volume reserve capacity between animals. Another possible explanation for the observed differences in intracranial elastance between animals is that these reflect limitations of the methodology that may be due to measurement error or the effect of another unknown parameter. In this initial small study, it is not possible to determine whether the observed variance between animals is due to true differences in intracranial volume reserve capacity. Only larger studies in large-animal models and eventually in patients will be able to provide insight into this key point. Future investigations may also seek to measure intracranial elastance in each hemisphere, both to ascertain the variability of measure, and to determine whether measurable differences in intracranial elastance exist between the hemispheres. Last, our current study was not specifically designed to identify elastance thresholds that may have clinical relevance, such as predicting the risk of herniation or of untoward ICP elevation. Further studies that seek to define relevant thresholds of intracranial elastance will be needed. Both the absolute value and rate of change of cardiac-gated intracranial elastance measures that may serve as markers of clinical deterioration await further investigations.

\section{Conclusions}

Measuring cardiac-gated elastance in a large-animal model of cerebral edema is feasible and measurements differ based on their relation to the cardiac cycle. Cardiacgated elastance rises progressively as cerebral edema and ICP increase, but the pattern and rate of rise may differ between swine. Paired ICP-elastance measurements may offer an improved physiological marker of intracranial volume reserve and enhance the ability to predict the risk of impending cerebral herniation.

\section{Acknowledgments}

This study was supported by a KAMIN research grant (KAMIN Grant \#56350) issued by the Israeli Innovation Authority and awarded to Dr. Doron and Prof. Barnea.

\section{References}

1. Kellie G. An account of the appearance observed in the dissection of two or three individuals presumed to have perished in the storm of the third and whose bodies were discovered in the vicinity of Leith on the morning of the fourth November 1821, with some reflections on the pathology of the brain. Trans Med Chir Soc Edinb. 1824;I:84-169.

2. Monro A. Observations on the Structure and Function of the Nervous System. Creech \& Johnston; 1783.

3. Langfitt TW, Weinstein JD, Kassell NF. Cerebral vasomotor paralysis produced by intracranial hypertension. Neurology. 1965;15:622-641.

4. Marmarou A, Shulman K, LaMorgese J. Compartmental analysis of compliance and outflow resistance of the cerebrospinal fluid system. J Neurosurg. 1975;43(5):523-534.

5. Marmarou A, Shulman K, Rosende RM. A nonlinear analysis of the cerebrospinal fluid system and intracranial pressure dynamics. J Neurosurg. 1978;48(3):332-344.

6. Miller JD, Garibi J, Pickard JD. Induced changes of cerebrospinal fluid volume. Effects during continuous monitoring of ventricular fluid pressure. Arch Neurol. 1973;28(4):265-269. 
7. Miller JD, Garibi J. Intracranial volume/pressure relationships during continuous monitoring of ventricular fluid pressure. In: Brock M, Dietz H, eds. Intracranial Pressure. Springer; 1972:270-274.

8. Miller JD. Volume and pressure in the craniospinal axis. Clin Neurosurg. 1975;22:76-105.

9. Miller JD, Pickard JD. Intracranial volume pressure studies in patients with head injury. Injury. 1974;5(3):265-268.

10. Hase U, Reulen HJ, Meinig G, Schürmann K. The influence of the decompressive operation on the intracranial pressure and the pressure-volume relation in patients with severe head injuries. Acta Neurochir (Wien). 1978;45(1-2):1-13.

11. Sullivan HG, Miller JD, Griffith RL III, Becker DP. CSF pressure-volume dynamics in neurosurgical patients: a preliminary evaluation in six patients. Surg Neurol. 1978;9(1):47-54.

12. Sullivan HG, Miller JD, Griffith RL III, et al. Bolous versus steady-state infusion for determination of CSF outflow resistance. Ann Neurol. 1979;5(3):228-238.

13. Heldt T, Zoerle T, Teichmann D, Stocchetti N. Intracranial pressure and intracranial elastance monitoring in neurocritical care. Annu Rev Biomed Eng. 2019;21:523-549.

14. Ramirez de Noriega F, Manley GT, Moscovici S, et al. A swine model of intracellular cerebral edema - cerebral physiology and intracranial compliance. J Clin Neurosci. 2018;58:192-199.

15. Doron O, Or T, Battino L, et al. Cerebral blood flow augmentation using a cardiac-gated intracranial pulsating balloon pump in a swine model of elevated ICP. J Neurosurg. 2020;132(5):1606-1615.

16. Leech P, Miller JD. Intracranial volume-pressure relationships during experimental brain compression in primates. 1 . Pressure responses to changes in ventricular volume. J Neurol Neurosurg Psychiatry. 1974;37(10):1093-1098.

17. Stocchetti N, Mattioli C, Mainini P, et al. Clinical use of cerebral elastance and intracranial dynamics measurements. Article in Italian. Minerva Anestesiol. 1993;59(1-2):1-9.

18. Abdullah J, Zamzuri I, Awang S, et al. Preliminary report on Spiegelberg pre and post-operative monitoring of severe head-injured patients who received decompressive craniectomy. Acta Neurochir Suppl. 2005;95:311-314.

19. Piper I, Spiegelberg A, Whittle I, et al. A comparative study of the Spiegelberg compliance device with a manual volumeinjection method: a clinical evaluation in patients with hydrocephalus. Br J Neurosurg. 1999;13(6):581-586.

20. Yau YH, Piper IR, Contant C, et al. Assessment of different data representations and averaging methods on the Spiegelberg compliance device. Acta Neurochir Suppl. 2005;95:289_ 292.

21. Anile C, Portnoy HD, Branch C. Intracranial compliance is time-dependent. Neurosurgery. 1987;20(3):389-395.

22. Bratton SL, Chestnut RM, Ghajar J, et al. Guidelines for the management of severe traumatic brain injury. VIII. Intracranial pressure thresholds. J Neurotrauma. 2007;24(suppl 1):S55-S58. Published correction appears in J Neurotrauma. 2008;25(3):276-278.

23. Carney N, Totten AM, O'Reilly C, et al. Guidelines for the Management of Severe Traumatic Brain Injury, Fourth Edition. Neurosurgery. 2017;80(1):6-15.
24. Hutchinson PJ, Kolias AG, Timofeev IS, et al. Trial of decompressive craniectomy for traumatic intracranial hypertension. N Engl J Med. 2016;375(12):1119-1130.

25. Lazaridis C, DeSantis SM, Smielewski P, et al. Patient-specific thresholds of intracranial pressure in severe traumatic brain injury. J Neurosurg. 2014;120(4):893-900.

26. Sheth KN, Stein DM, Aarabi B, et al. Intracranial pressure dose and outcome in traumatic brain injury. Neurocrit Care. 2013;18(1):26-32.

27. Vik A, Nag T, Fredriksli OA, et al. Relationship of "dose" of intracranial hypertension to outcome in severe traumatic brain injury. J Neurosurg. 2008;109(4):678-684.

28. Chesnut R, Videtta W, Vespa P, Le Roux P. Intracranial pressure monitoring: fundamental considerations and rationale for monitoring. Neurocrit Care. 2014;21(suppl 2):S64-S84.

29. Chesnut RM. A conceptual approach to managing severe traumatic brain injury in a time of uncertainty. Ann N Y Acad Sci. 2015;1345:99-107.

30. Di Rocco C, Pettorossi VE, Caldarelli M, et al. Experimental hydrocephalus following mechanical increment of intraventricular pulse pressure. Experientia. 1977;33(11):1470-1472.

\section{Disclosures}

Prof. Barnea and Dr. Doron are listed as inventors on a patent of the intracranial cardiac-gated balloon pump used in this work. Prof. Barnea is the founder and CEO of Syncath Neuroscience. Drs. Nossek, Rosenthal, and Stocchetti serve as scientific advisors on the medical advisory board for Syncath, Ltd.

\section{Author Contributions}

Conception and design: Rosenthal, Doron, Barnea. Acquisition of data: Rosenthal, Doron, Or. Analysis and interpretation of data: Rosenthal, Doron, Barnea, Stocchetti. Drafting the article: Rosenthal, Doron. Critically revising the article: Rosenthal, Doron, Barnea, Stocchetti, Nossek. Reviewed submitted version of manuscript: all authors. Approved the final version of the manuscript on behalf of all authors: Rosenthal. Statistical analysis: Doron. Administrative/technical/material support: Rosenthal, Barnea, Or, Nossek. Study supervision: Rosenthal, Barnea.

\section{Supplemental Information \\ Previous Presentations}

Preliminary data from this work were presented as an oral presentation at the Annual Meeting of the Israeli Neurosurgical Society, May 2019, Upper Galilee, Israel, and as an oral presentation at the International Symposium on Intracranial Pressure and Neuromonitoring (ICP) 2019 meeting in Leuven, Belgium, September 2019.

\section{Correspondence}

Guy Rosenthal: Hadassah-Hebrew University Medical Center, Kiryat Hadassah, Jerusalem, Israel. rosenthalg@hadassah.org.il. 